\title{
Study of dam break flow interaction with urban settlements over a sloping channel.
}

\author{
Ioanna Stamataki ${ }^{1,}$, Jun Zang ${ }^{1}$, Eugeny Buldakov ${ }^{2}$, Thomas Kjeldsen ${ }^{1}$, and Dimitris \\ Stagonas $^{2}$ \\ ${ }^{1}$ Research Unit for Water, Environment and Infrastructure Resilience (WEIR), Department of \\ Architecture and Civil Engineering, University of Bath, Claverton Down Road, Bath, BA2 7AY, UK \\ ${ }^{2}$ Department of Civil, Environmental and Geomatic Engineering, University College London, Gower \\ Street, London, WC1E 6BT, UK
}

\begin{abstract}
This paper describes a dam break experiment on a sloped channel, carried out in a hydraulic flume at UCL for the purpose of computer model validations of extreme events, such as flash floods. An elevated reservoir was situated upstream followed by a $1 / 20$ slope leading up to a flat floodplain. Plexiglas blocks were positioned on the floodplain constituting different urban settlements and creating different obstructions to the flow. The flume was instrumented along its length measuring the change in water depth in the reservoir; the water depth time histories in various locations; the flow patterns and flood front velocity; and lastly the pressure and load on the buildings. The experiments were repeated for different urban settlements, flood intensities (two different initial water depths in the reservoir) and roughness layers along the slope, representative of a vegetated and a non-vegetated hill. In the present study, the experimental results were described qualitatively and compared with theoretical processes and 2D numerical results obtained using OpenFOAM's RAS turbulent model. Water depth, velocity and load measurements were analysed for different cases and it was found that while the 2D model provided a good fit on the slope, the flows generated around the building were more complex 3D formations which lead to inaccuracies. All experiments were repeated multiple times to ensure repeatability and thus the procedure was validated successfully providing a complete dataset that can be used for the validation of computational models for extreme events.
\end{abstract}

\section{Introduction}

One expected consequence of climate change is an increase in the frequency and magnitude of flooding. 21 million people globally are being threatened by it each year and this number is expected to rise to 54 million by 2030 [1,2]. Flash floods are floods most often caused by a short period intense rainfall [3] and are a destructive natural hazard with one of the highest mortalities. Small catchments often have a naturally flashy response to intense rainfall resulting in important damage from small localised events [4]. Examples in the UK include the Boscastle flash flood in 2004, the 2005 event in Helmsley and the 1952 flash flood in

\footnotetext{
${ }^{\star}$ Corresponding author: i.stamataki@ bath.ac.uk
} 
Lynmouth [5]. Flash floods remain a global problem and due to their dynamic nature combined with their limited spatial and temporal scales, observation and accurate modelling of these events continue to be a challenge. As very limited field data exist from flash floods, a practical approach to initially generate flash floods, both numerically and experimentally, is by representing them by a dam break failure. This guarantees their main characteristic features [3]: rapidity of onset and the rate of rise in level.

The dam break problem has become a widely researched problem and modelled both experimentally and numerically. Research started as early as 1960 with [6] the US Army Engineers Waterways Experiment Station publishing a report on experimental cases on floods resulting from suddenly breached dams. The research continued from simple experimental studies such as the initial stages of a dam-break [7] to more complicated problems such as dam-break induced mudflows [8]. Numerically, the dam-break problem has been modelled in 1D, 2D and 3D [9, 10] and experimental and numerical results have been compared by several researchers $[8,11]$. However, a report published by Defra and the Environment Agency [12] showed that while most industry models provide an appropriate support tool for decision making in flood risk management, they do not provide enough precision when dam breaks are modelled, specifically regarding the detail of the transition from supercritical to subcritical flow.

There has been a limited number of publications on hydraulic models specifically designed to represent flash floods. In a laboratory setting the most known flash flood experiment is the Testa et al. experiment which was part of the IMPACT project, a project assessing the risks from extreme flooding [13]. Numerically, flash floods have been modelled either with distributed hydrological models looking into runoff [14], or with hydrodynamic models examining the dynamic routing of the flood wave and the water levels within the simulation domain [15]. Thus, the aim of this experimental work was to generate flash floods in a controlled laboratory environment on a sloping channel in order to provide further physical understanding of flash floods and their impact, and use the data to validate the numerical simulations of selected numerical hydrodynamic modelling systems. In this paper the experimental results will be compared with numerical results from OpenFOAM.

The structure of this paper is as follows. First, Section 2 describes the experimental setup and experimental cases. Next, Section 3 presents a qualitative description of the results and Section 4 explains the numerical model used. Then, in Section 5 experimental results are presented for no building and one building cases and compared with numerical results. Finally, Section 6 discusses results and draws concluding remarks.

\section{Description of experiments}

The experiment was conducted in University College London's, United Kingdom (UCL) flume (Mechanical Engineering Department), which is 20 meters long and 1.2 meters wide. An elevated reservoir was built at the upstream end of the flume, separating the reservoir and the floodplain (Fig.1) and the downstream boundary was free created by a step for discharge.

\begin{tabular}{|c|c|c|c|c|}
\hline Reservoir - & $\mathbb{D}_{\longrightarrow}^{\text {Gate }}$ & & & \\
\hline 0.3 & & 1/20 Slope & 0.2 & \\
\hline 2.9 & & 6.0 & 0.20 .7 & 2.1 \\
\hline
\end{tabular}

Fig. 1. Sketch of experimental set-up (all dimensions in meters) 
The dam was $2.9 \mathrm{~m} \times 1.2 \mathrm{~m}$ with one hinged gate along its width allowing it to rotate upwards (Fig. 2,3). This resulted in an accelerated flow at the bottom due to the pressure drop allowing us to mimic instantaneous release. The gate rotated upwards using a weight system and in order to assess if its release was instantaneous, two time criteria from literature $t_{o p 1}$ and $t_{o p 2}$ were used, where $t_{o p}$ is the time it takes the gate to open and $h_{o}$ is the initial water depth $\left(h_{o}=0.2\right.$ and $h_{o}=0.1$ ). The first criterion is $t_{o p 1} \leq 1.25 \sqrt{h_{o} / g}[16]$ for which $t_{o p 1_{0} 0.2}=0.178 \mathrm{~s}$ and $t_{o p 1 \_0.1}=0.126 s$ for $\mathrm{H} 200$ and $\mathrm{H} 100$ respectively. The second criterion is $t_{o p 2} \leq \sqrt{2 h_{o} / g}$ [17] for which $t_{o p 2 \_0.2}=0.202 s$ and $t_{o p 2 \_0.1}=0.142 s$ for $\mathrm{H} 200$ and $\mathrm{H} 100$ respectively. The experiment resulted in opening times $t_{o p_{-} 0.2}=0.172 s$ and $t_{o p_{-} 0.1}=0.124 s$ for $\mathrm{H} 200$ and $\mathrm{H} 100$ respectively thus satisfying both criteria and confirming the release was instantaneous.

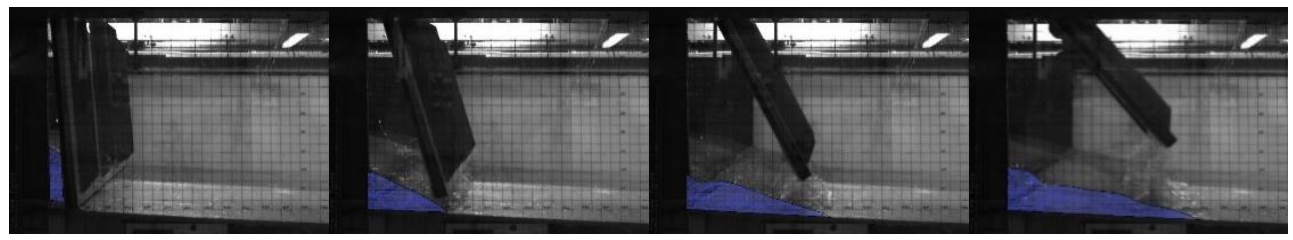

Fig. 2. Gate opening for $h_{o}=0.2 m$. From left to right, $\mathrm{t}=0.08 \mathrm{~s}, \mathrm{t}=0.172 \mathrm{~s}, \mathrm{t}=0.22 \mathrm{~s}$ and $\mathrm{t}=0.28 \mathrm{~s}$

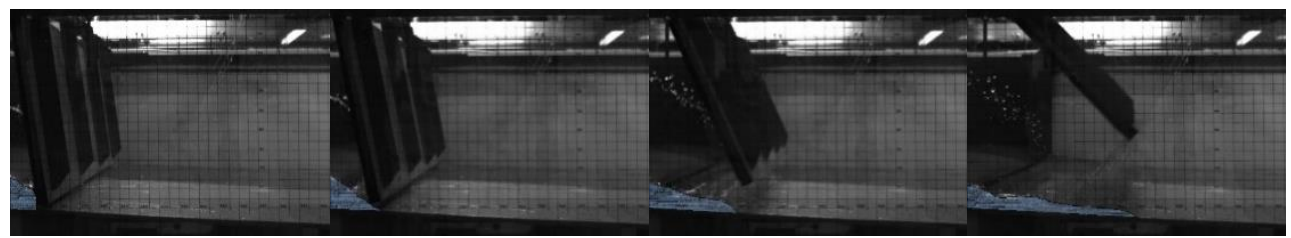

Fig. 3. Gate opening for $h_{o}=0.1 \mathrm{~m}$. From left to right, $\mathrm{t}=0.1 \mathrm{~s}, \mathrm{t}=0.124 \mathrm{~s}, \mathrm{t}=0.2 \mathrm{~s}$ and $\mathrm{t}=0.28 \mathrm{~s}$

As part of this project, 18 test cases were examined considering: two initial water levels in the reservoir, different roughness (un-vegetated smooth slope and vegetated), different densities of the urban texture (none, one, two and four buildings). Table 1 presents the selected test cases for the purpose of this paper:

Table 1. Experimental set up test cases.

\begin{tabular}{|l|l|l|l|l|}
\hline Set & Initial water depth $[\mathbf{m}]$ & Roughness layer & Building(s) & Name \\
\hline \multirow{2}{*}{ Set 01 } & 0.2 & No & None & B0 H200 \\
\cline { 2 - 5 } & 0.2 & No & Single & B1 H200 \\
\hline \multirow{2}{*}{ Set 02 } & 0.1 & No & None & B0 H100 \\
\cline { 2 - 5 } & 0.1 & No & Single & B1 H100 \\
\hline \multirow{2}{*}{ Set 03 } & 0.2 & Yes & None & B0 H200G \\
\cline { 2 - 5 } & 0.2 & Yes & Single & B1 H200G \\
\hline \multirow{2}{*}{ Set 04 } & 0.1 & Yes & None & B0 H100G \\
\cline { 2 - 5 } & 0.1 & Yes & Single & B1 H100G \\
\hline
\end{tabular}

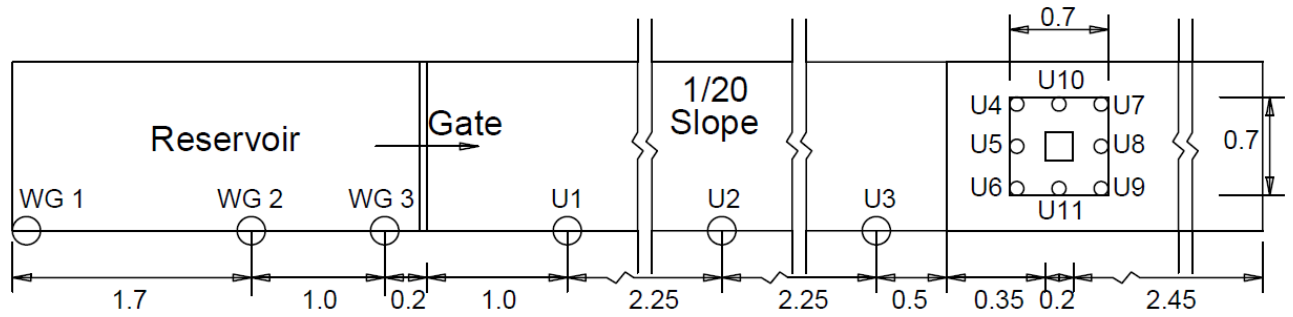

Fig. 4. Wave gauges and ultrasonic sensor positions

The flume and buildings were instrumented along their length with wave gauges; ultrasonic sensors; high speed cameras; pressure sensors and load cells. Firstly, three wave probes were installed along the reservoir's length measuring the change in water depth (see Fig. 4). Then, 
ultrasonic sensors were positioned in 11 different locations (see Fig. 4) recording the water depth time histories with a minimum distance of $0.2 \mathrm{~m}$ from the building (see Fig. 5).

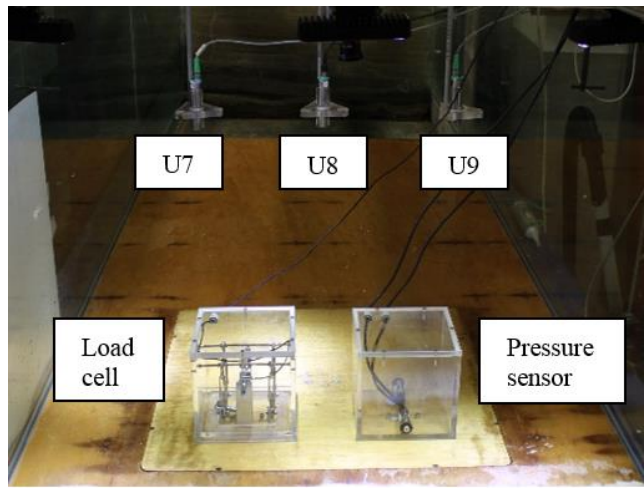

Fig. 5. Ultrasonic and pressure sensors and load cell
Furthermore, load cells were used to measure the load time histories along the x-axis. Additionally, pressure on the buildings was measured using pressure sensors installed inside the blocks. Finally, the flow patterns and flood front velocity were recorded using two Mako-G high speed cameras (250fps) placed above and on the side of the flume. Multiple repetitions were performed for all experimental test cases ensuring repeatability and validation of the procedure.

\section{Description of results}

In 1892 Ritter [18] contributed to the dam break problem with a landmark theory on the idealised dam break, solving the dam break wave shape [19]. His theory, describes a dam break in a dry channel where the dam has an initial water depth $h_{o}$ and is separated from the rest of the channel by suddenly removed wall. As soon as the wall is removed, a dam break wave starts moving downstream and a negative wave starts propagating upstream within the reservoir [18]. Fig. 6, shows the decrease in water depth in the reservoir over time from the three wave gauges (refer to Fig. 4).
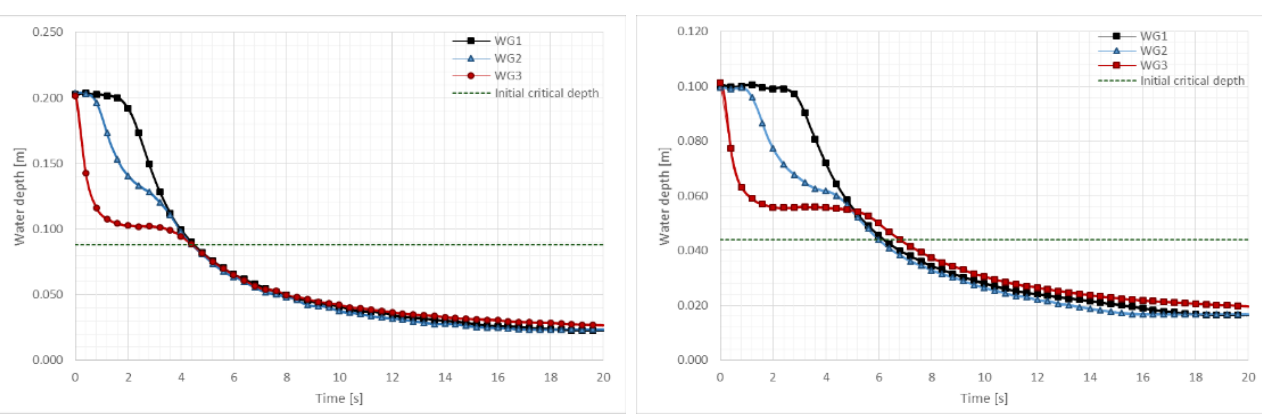

Fig. 6. Decrease of water depth over time in reservoir for $\mathrm{H}=0.2 \mathrm{~m}$ and $\mathrm{H}=0.1 \mathrm{~m}$ respectively

Ritter's equation $x / t=2 \sqrt{g h_{o}}-3 \sqrt{g h}$ [18] describes the surface profile between the dam break front and the negative wave, where $h_{o}$ is the initial water depth and $h$ is the depth at $x$ for time $t$. Critical flow conditions are observed at the origin when the specific energy is minimum and a constant depth is predicted [19]. Analysing the results from the three wave gauges in the reservoir (Fig. 7) a good agreement is found between theoretical and experimental results. WG1 in both cases does not provide a good fit but this is expected due to its location being so close to the back wall of the reservoir. WG2 and WG3 show very good agreement especially up to 4 and 5 seconds for the H200 and H100 case respectively. Possible reasons for these differences are firstly the gate release which is not as instantaneously as in theory, resulting in higher water depths experimentally. Secondly, as soon as the gate is released the water is moving under the influence of gravity and created waves, may demonstrate wave dispersion. 


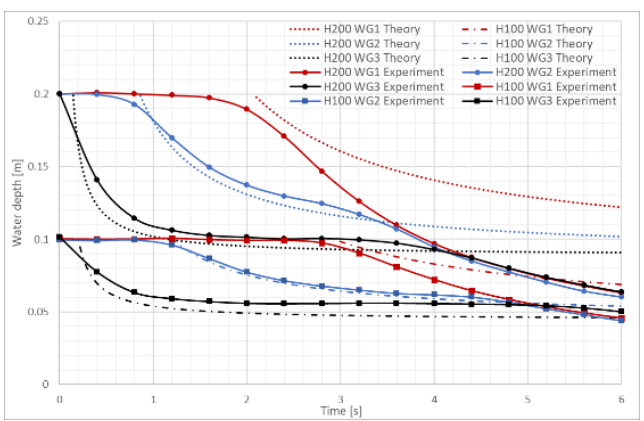

Fig. 7. Water depths reservoir
Nevertheless, the experimental results lead to a higher water depth in average by $0.009 \mathrm{~m}$ for $\mathrm{H} 200$ and $0.013 \mathrm{~m}$ for $\mathrm{H} 100$ equivalent to $4.84 \%$ and $16.8 \%$ respectively. Therefore, the experiment was considered to follow the ideal dam break theory and using Ritter's equations [18] the theoretical initial critical depth was calculated and plotted on the different plots $h_{(x=0)}=4 / 9 h_{o}=0.088$ for $h_{o}=0.2 m$ and $h_{(x=0)}=4 / 9 h_{o}=0.044$ for $h_{o}=0.1 m[19]$.

The negative wave has a slope $d t / d x=1 / C_{o}$ where $C_{o}=\sqrt{g h_{o}}$ [18]. On the slope, the water depth $h_{o}$ is at all times smaller than the initial critical depth $h_{o}<h_{c}$, (Fig. 8) resulting in a supercritical flow [19]. Fig. 8 shows the evolution of the water depth over time for three different locations along the slope. Furthermore, the gap between measurements when comparing the vegetated and the non-vegetated smooth slope, increases further down the slope as the velocity decrease is more apparent. Between U1 and U3, it is 1.2 and 1.37 times slower with grass than without grass for $\mathrm{H} 200$ and $\mathrm{H} 100$ respectively showing that the change in roughness has a more important effect in lower water depths and velocities.
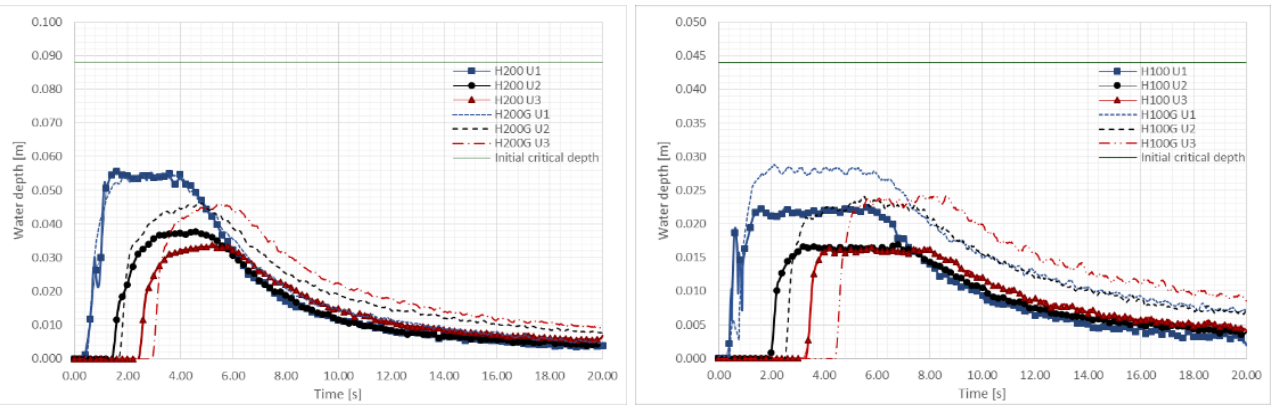

Fig. 8. Water depths at U1, U2 and U3 along the slope for $\mathrm{H} 200$ and $\mathrm{H} 100$ respectively with (H200G, H100G) and without grass (H200, H100)
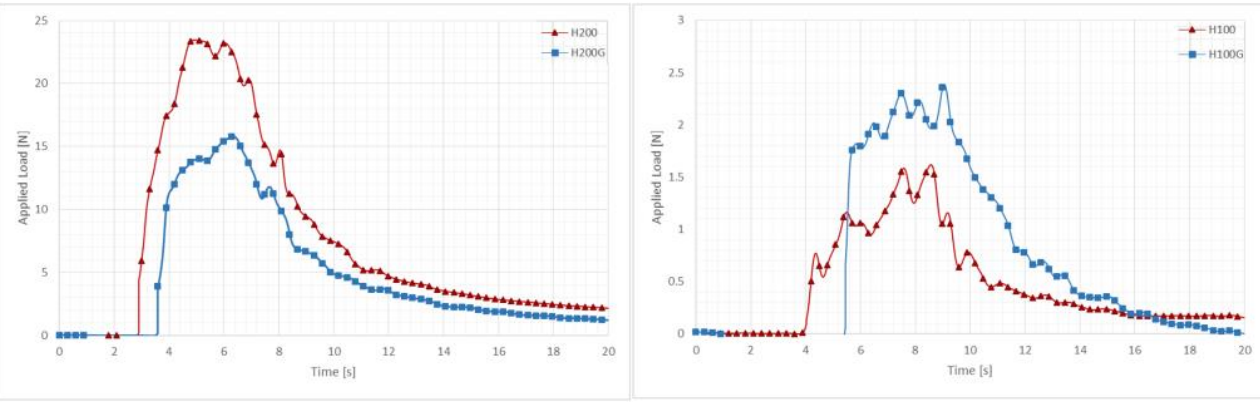

Fig. 9. Applied load over time for H200, H200G and H100, H100G respectively

The impact on the downstream settlements is based on the theory of an object in supercritical flow and can be described in three stages: impact state, decrease, and stabilisation. Fig. 9 shows the applied load over time for the different cases. The vegetated slope decreases the peak for H200 but creates a higher peak in the H100 case. For H200 the impact state is represented up to 6 seconds, then the decrease happens between 6 and 11 seconds and the 
stabilisation is apparent after 11 seconds. Respectively for H100 the impact state is up to 8 seconds, then the decrease between 8 and 13 seconds and the stabilisation after 13 seconds.

\section{Numerical model}

OpenFOAM was developed in the 1980s and released as an open-source software in 2004. It is a C++ toolbox used for solving computational fluid dynamic problems [20]. The multiphase solver interFoam models the interface between the water and air, it solves the Navier-Stokes equations and to record the position of the water/air interface. It uses the VoF method (Volume of Fluid). Here, a turbulence model was used, the Reynolds-averaged simulation (RAS) model. The standard k- $\varepsilon$ model, uses a transport equation and solves for the kinetic energy $k$ and turbulent dissipation $\varepsilon$ [21], which is the rate at which the energy created from turbulence is dissipated [22]. The numerical model replicating the experimental set up was designed as a 2D model. The model was initially calibrated and the computational mesh was represented by a rectangular domain resulting to 202813 points representative of a 0.002 global mesh. Boundary conditions allocated for all variables can be found on Table 2 . The time step of the simulations was controlled by maintaining the Courant condition using an adaptive time step to satisfy two requirements: maxAlphaCo and maxCo equal to 1 [22].

\begin{tabular}{|l|l|l|l|l|l|}
\hline \multicolumn{4}{|c}{ Table 2. Numerical model parameters } & \\
\hline Variable & Inlet & Outlet & Top & Bottom \\
\hline alpha & zeroGradient & inletOutlet & inletOutlet & zeroGradient \\
\hline Velocity & noSlip & pressureInletOutletVelocity & pressureInletOutletVelocity & noSlip \\
\hline Pressure & fixedFluxPressure & totalPressure & totalPressure & fixedFluxPressure \\
\hline $\boldsymbol{k}$ & kqRWallFunction & inletOutlet & inletOutlet & empty \\
\hline epsilon & epsilonWallFunction & inletOutlet & inletOutlet & empty \\
\hline
\end{tabular}

\section{Analysis and discussion}

In this section, the experimental measurements (water depths, velocities, loads) are compared with the numerical results at representative locations. First the velocity is compared for the different test cases, then the water depths at positions U1, U2 and U3 and U5 and finally the load acting on the buildings' surface.

The experimental front velocity was found to be $2.163 \mathrm{~m} / \mathrm{s}$ for $\mathrm{H} 200$ compared to $2.189 \mathrm{~m} / \mathrm{s}$ in the numerical simulation, for $\mathrm{H} 100$ the observed velocity was $1.642 \mathrm{~m} / \mathrm{s}$ compared to $2.304 \mathrm{~m} / \mathrm{s}$ numerically, and finally $1.793 \mathrm{~m} / \mathrm{s}$ for $\mathrm{H} 200 \mathrm{G}$ and $1.194 \mathrm{~m} / \mathrm{s}$ for H100G. The numerically modelled velocity along the slope for $\mathrm{H} 200$ was therefore over predicted by $1.2 \%$ showing a very good agreement with the experimental data. In contrast, the water depth was underestimated by $20 \%$ for $\mathrm{H} 100$ as in lower speeds the roughness layer had more effect.
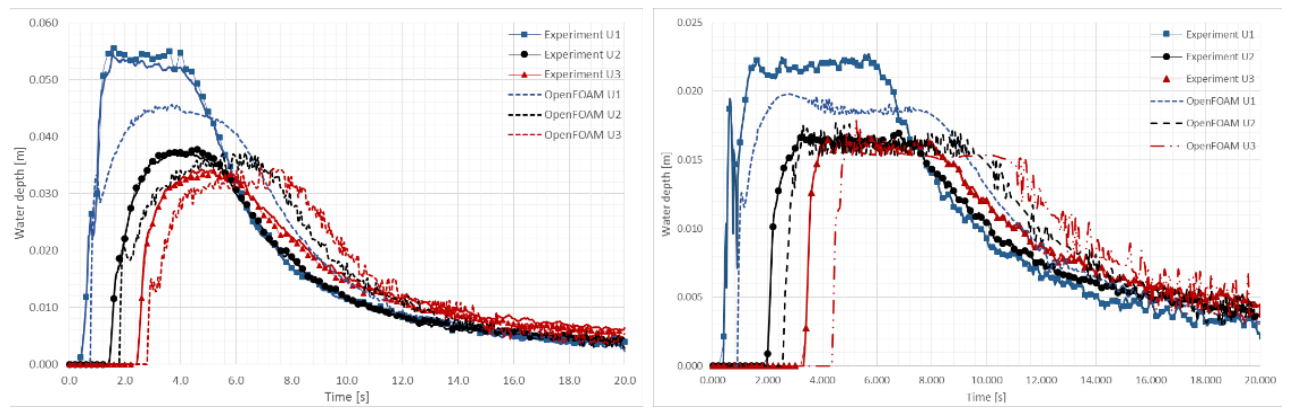

Fig. 10. Comparison of experimental with numerical results for water depths for H200 and H100 respectively for positions $\mathrm{U} 1, \mathrm{U} 2$ and $\mathrm{U} 3$ 
Fig. 10 shows the comparison of experimental and numerical results for water depth over time at the three positions on the slope U1, U2 and U3 for H200 (left) and H100 (right). For both cases there is a delay in the OpenFOAM simulation which is larger for H100 due to the lower velocities. In both cases the peak at U1 location is underestimated contrary to the peaks in U2 and U3 which are in closer agreement. Nonetheless, the decrease stage is over estimated in all cases before the stabilisation stage where in all cases experimental and numerical results are in agreement.

Finally, Fig. 11 shows the comparison of experimental and numerical results for water depths over time at position U5, in front of the building, for B0 and B1. Looking only at the impact stage, it is apparent that the B0 case is better represented by the numerical simulation. On the other hand, the B1 case where the physical characteristics and flow formations are strongly $3 \mathrm{D}$, the numerical simulation does not provide such an accurate representation. It results in a much higher peak as the building creates blockage not allowing an alternative escape route for the water.
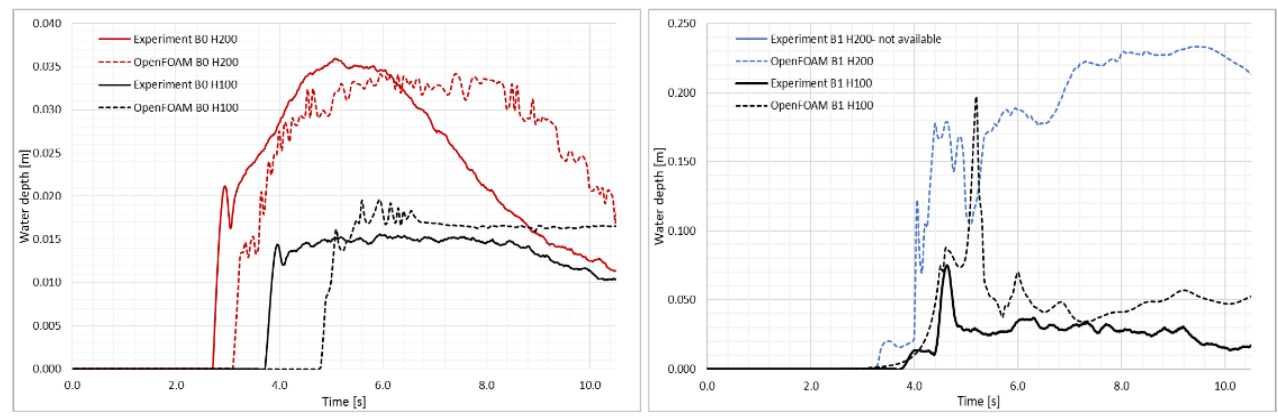

Fig. 11. Comparison of experimental with numerical results for water depths for $\mathrm{H} 200$ and $\mathrm{H} 100$ at position U5 for no building (B0) and one building (B1) respectively

\section{Conclusions}

Experimental results were presented from a flash flood experiment in a controlled environment. A qualitative description of the experiment and data was provided emphasising the theoretical processes apparent from the gate release to the impact stages in the built environment. Using OpenFOAM, experimental and numerical results were compared providing a good agreement on the water depth and velocity calculations along the slope. Overall, this paper shows that the experiments provide a valuable dataset that can be used for the validation of computational models for extreme events. The RAS OpenFOAM model provided a good approximation especially in the water depths along the slope but as most physical characteristics of these flows are three-dimensional, the agreement was not as good in the downstream part after the slope. Consequently, this encourages further investigations of the physical processes of flash floods and the interaction between structures with flash flood waves. Future work will include convergence tests, further 2D models of the slope and $3 \mathrm{D}$ modelling of the floodplain using different turbulence models for comparative reasons.

The authors would like thank the WISE EPSRC grant (Grant EP/L016214/1) for supporting this research, Balena High Performance Computing (HPC) Service at the University of Bath and the support of the Department of Civil, Environmental and Geomatic Engineering at UCL for their involvement, advice and irreplaceable help. 


\section{References}

1. World Resources Institute. ACT 2015 Publications. 2015 [cited 201627 January]; Available from: http://www.wri.org/our-work/project/act-2015/publications.

2. An, H. and S. Yu, Well-balanced shallow water flow simulation on quadtree cut cell grids. Advances in Water Resources, 2012. 39: p. 60-70.

3. Archer, D.R. and H.J. Fowler, Characterising flash flood response to intense rainfall and impacts using historical information and gauged data in Britain. Journal of Flood Risk Management, 2015: p. n/a-n/a.

4. Werner, M. and M. Cranston, Understanding the Value of Radar Rainfall Nowcasts in Flood Forecasting and Warning in Flashy Catchments. Meteorological Applications, 2009. 16(1): p. 41-55.

5. Defra; Environment Agency, Understanding of and response to severe flash flooding 2009: Bristol.

6. Corps of Engineers, Flood Resulting from suddenly breached dams. 1960: Mississippi.

7. Stansby, P.K., A. Chegini, and T.C.D. Barnes, The initial stages of dam-break flow. Journal of Fluid Mechanics, 1998. 374: p. 407-424.

8. Peng, S.H. and S.C. Chen, Comparison of numerical and experimental study of dambreak induced mudflow. Sediment Dynamics and the Hydromorphology of Fluvial Systems, 2006. 306: p. 548-555.

9. Zhainakov, A.Z. and A.Y. Kurbanaliev, Verification of the open package OpenFOAM on dam break problems. Thermophysics and Aeromechanics, 2013. 20(4): p. 451-461.

10. Marsooli, R. and W. Wu, 3-D finite-volume model of dam-break flow over uneven beds based on VOF method. Advances in Water Resources, 2014. 70: p. 104-117.

11. Aureli, F., et al., Experimental and numerical evaluation of the force due to the impact of a dam-break wave on a structure. Advances in Water Resources, 2015. 76: p. 29-42.

12. Defra and Environment Agency, Benchmarking of 2D Hydraulic Modelling Packages, in Flood and Coastal Erosion Risk Management Research and Development Programme. 2010: Bristol.

13. Testa, G., et al., Flash flood flow experiment in a simplified urban district. Journal of Hydraulic Research, 2007. 45(Extra issue): p. 37-44.

14. Braud, I., et al., The use of distributed hydrological models for the Gard 2002 flash flood event: Analysis of associated hydrological processes. Journal of Hydrology, 2010. 394(1-2): p. 162-181.

15. Huang, W., et al., Full 2D hydrodynamic modelling of rainfall-induced flash floods. Journal of Mountain Science, 2015. 12(5): p. 1203-1218.

16. Vischer, D. and W.H. Hager, Dam hydraulics. 1998: Wiley.

17. Lauber, G. and W.H. Hager, Experiments to dambreak wave: Horizontal channel. Journal of Hydraulic Research, 1998. 36(3): p. 291-307.

18. Ritter, A., Die Fortpflanzung der Wasserwellen. . Vereine Deutcher Ingenieure Zeitswchrift, 1892. 36: p. 947-954.

19. Chanson, H., Hydraulics of Open Channel Flow. Second ed. 2004.

20. Damián, S.M., Description and utilization of interFoam multiphase solver. 2012, International Center for Computational Methods in Engineering.

21. Furbo, E., Evaluation of RANS turbulence models for flow problems with significant impact of boundary layers 2010, Uppsala Universitet. p. 56.

22. Greenshields, C.J., OpenFOAM:The Open Source CFD Toolbox, User Guide. 2015. 